\title{
Potentially Inappropriate Use of Intravenous Opioids in Hospitalized Patients
}

\author{
Amber B Moore, MD, MPH ${ }^{1,2 \star}$, Scott Navarrett, MD ${ }^{1,2}$, Shoshana J Herzig, MD, MPH ${ }^{1,2}$
}

'Division of General Medicine, Department of Medicine, Beth Israel Deaconess Medical Center, Boston, Massachusetts; ${ }^{2}$ Harvard Medical School, Boston, Massachusetts.

Physicians have the potential to decrease opioid misuse through appropriate prescribing practices. We examined the frequency of potentially inappropriate intravenous (IV) opioid use (where oral use would have been more appropriate) in patients hospitalized at a tertiary medical center. We excluded patients with cancer, patients receiving comfort care, and patients with gastrointestinal dysfunction. On the basis of recent guidance from the Society of Hospital Medicine, we defined IV doses as potentially inappropriate if administered more than 24 hours after an initial IV dose in patients who did not have nil per os status. Of the 200 patients studied, $31 \%$ were administered potentially inappropriate IV opioids at least once during their hospitalization, and $33 \%$ of all IV doses administered were potentially inappropriate. Given the numerous advantages of oral over IV opioids, this study suggests significant potential for improving prescribing practices to decrease risk of addiction, costs, and complications, ultimately improving the value of care provided. Journal of Hospital Medicine 2019;14:678-680. @ 2019 Society of Hospital Medicine

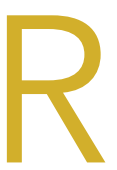

ecently released guidelines on safe opioid prescribing draw attention to the fact that physicians have the ability to curb the opioid epidemic through better adherence to prescribing guidelines and limiting opioid use when not clinically indicated. ${ }^{1,2} \mathrm{~A}$ consensus statement from the Society of Hospital Medicine includes 16 recommendations for improving the safety of opioid use in hospitalized patients, one of which is to use the oral route of administration whenever possible, reserving intravenous (IV) administration for patients who cannot take food or medications by mouth, patients suspected of gastrointestinal (GI) malabsorption, or when immediate pain control and/or rapid dose titration is necessary. ${ }^{2}$ This recommendation was based on an increased risk of side effects, adverse events, and medication errors with IV compared with oral formulations. ${ }^{3-5}$ Furthermore, the reinforcement from opioids is inversely related to the rate of onset of action, and therefore opioids administered by an IV route may be more likely to lead to addiction..$^{6-8}$

Choosing oral over IV opioids has several additional advantages. The cost of the IV formulation is more than oral; at our institution, the cost of IV morphine is 2.5-4.6 times greater than oral. Additional costs associated with IV administration include nursing time and equipment. Overall, transitioning patients from IV to oral medications could considerably lower costs of care. ${ }^{9}$ Ongoing need for an IV line may also lead to avoidable complications, including patient discomfort, infection, and thrombophlebitis. In addition, the recent national shortage of

*Corresponding Author: Amber Moore, MD, MPH; E-mail: abmoore@bidmc. harvard.edu; Telephone: 617-754-4677.

Published online first June 7, 2019

Received: December 27, 2018; Revised: March 26, 2019; Accepted: April 7, 2019

(c) 2019 Society of Hospital Medicine DOI 10.12788/jhm.3225
IV opioids has necessitated better stewardship of IV opioids.

Despite this recommendation, our observations suggest that patients often continue receiving IV opioids longer than clinically indicated. The goal of this study was to identify the incidence of potentially inappropriate IV opioid use in hospitalized patients.

\section{METHODS}

The present study was an observational study seeking to quantify the burden of potentially inappropriate IV opioid use and characteristics predicting potentially inappropriate use in the inpatient setting at a large academic medical center in Boston, Massachusetts, using retrospective review of medical records.

\section{Definition of Potentially Inappropriate Use and Study Sample}

We identified all hospitalizations during the month of February 2017 with any order for IV opioids using pharmacy charge data and performed chart reviews in this sample until we reached our prespecified study sample of 200 hospitalizations meeting inclusion/exclusion criteria further defined below.

We defined potentially inappropriate use of IV opioids as use of IV opioids for greater than 24 hours in a patient who could receive oral medications (evidenced by receipt of other orally administered medications during the same 24-hour period) and was not mechanically ventilated. This definition is consistent with recommendations in the recently released consensus statement from the Society of Hospital Medicine. ${ }^{2}$ We selected a time frame of 24 hours because IV pain medications may be indicated for initial immediate pain control and rapid dose titration; however, 24 hours should be sufficient time to determine opioid needs and transition to an oral regimen 
TABLE 1. Study Population Characteristics and Adjusted Associations with Any Potentially Inappropriate Opioid Use.

\begin{tabular}{|c|c|c|c|c|c|}
\hline Characteristic & Total Population & No Inappropriate Use & Any Inappropriate Use & $\begin{array}{c}\text { Bivariable } \\
P \text { Value }\end{array}$ & $\begin{array}{c}\text { Adjusted Relative Risk } \\
\text { (95\% Confidence Interval) }\end{array}$ \\
\hline $\mathrm{n}$ & 200 & 138 & 62 & & \\
\hline Age & & & & .19 & \\
\hline$<65$ & $134(67 \%)$ & $88(64 \%)$ & $46(74 \%)$ & & reference \\
\hline$>65$ & $66(33 \%)$ & $50(36 \%)$ & $16(26 \%)$ & & $0.55(0.27-1.10)$ \\
\hline Gender & & & & .65 & \\
\hline Male & $89(45 \%)$ & $63(46 \%)$ & $26(42 \%)$ & & reference \\
\hline \multirow[t]{2}{*}{ Female } & $111(56 \%)$ & $75(54 \%)$ & $36(58 \%)$ & & 1.18 \\
\hline & & & & & $(0.62-2.24)$ \\
\hline Ethnicity & & & & .06 & \\
\hline White & $135(68 \%)$ & $86(62 \%)$ & $49(79 \%)$ & & reference \\
\hline Black & $22(11 \%)$ & $17(12 \%)$ & $5(8 \%)$ & & $0.48(0.16-1.46)$ \\
\hline Other / unknown / patient declined & $43(22 \%)$ & $35(25 \%)$ & $8(13 \%)$ & & $0.39(0.17-0.93)$ \\
\hline Service & & & & .86 & \\
\hline Medical $^{\mathrm{a}}$ & $52(26 \%)$ & $35(25 \%)$ & $17(27 \%)$ & & reference \\
\hline Surgical ${ }^{b}$ & $148(74 \%)$ & $103(75 \%)$ & $45(73 \%)$ & & $0.78(0.38-1.60)$ \\
\hline Opioid drip/PCA use & & & & .14 & \\
\hline No & $169(84 \%)$ & $113(82 \%)$ & $56(90 \%)$ & & reference \\
\hline Yes & $31(16 \%)$ & $25(18 \%)$ & $6(10 \%)$ & & $0.40(0.15-1.09)$ \\
\hline
\end{tabular}

in patients without contraindications. After an initial IV dose, additional IV doses within 24 hours were considered appropriate, whereas IV doses thereafter were considered potentially inappropriate unless the patient had nil per os status, including medications. All IV opioids administered within 24 hours of a surgery or procedure were considered appropriate. Because it may be appropriate to continue IV opioids beyond 24 hours in patients with an active cancer diagnosis, in patients who have chosen comfort measures only, or in patients with Gl dysfunction (including conditions such as small bowel obstruction, colitis, pancreatitis), we excluded these populations from the study sample. Patients admitted to the hospital for less than 24 hours were also excluded from the study, because they would not be at risk for the outcome of potentially inappropriate use. Doses of IV opioids administered for respiratory distress were considered to be appropriate. Given difficulty in identifying the appropriate time to transition from patientcontrolled analgesia (PCA) to IV or per os (PO) opioids, days spent receiving opioids by PCA or continuous IV drip were excluded from the analysis.

We used Fisher's exact test or the Chi-square test (in the setting of a multicategory variable) to calculate bivariable $P$ values. We used multivariable logistic regression to identify independent predictors of receipt of at least one dose of potentially inappropriate IV opioids, using the hospitalization as the unit of analysis.

\section{RESULTS}

Of 630 hospitalizations with at least one order for IV opioids over a one-month period, we reviewed 502 charts, from which we excluded 76 hospitalizations with an active cancer diagnosis, 30 with comfort-focused care, 115 with Gl dysfunction, and 108 with a hospitalization less than 24 hours in duration, resulting in 200 hospitalizations included in this analysis (some patients met multiple exclusion criteria). Table 1 outlines characteristics of the study population, stratified by appropriateness of IV opioid use. The study population was predominately white and had an average age of 56.3 years. The majority of patients were on a surgical service. Hydromorphone was the most commonly administered opioid. There were significant differences in the percentage of doses considered inappropriate between different types of opioids $(P<.001)$, with morphine having the highest proportion of doses considered potentially inappropriate (Table 2).

Thirty-one percent of the cohort was administered at least one potentially inappropriate dose of IV opioids. A total of 432 of 1,319 (33\%) IV doses were considered potentially inappropriate.

\section{Predictors of Potentially Inappropriate Use}

No significant associations were observed between potentially inappropriate IV opioid administration and age, sex, or admitting service (Table 1). Patients with an ethnicity described as 
TABLE 2. Proportion of Potentially Inappropriate Opioid Administrations by Opioid Type

\begin{tabular}{|c|c|c|}
\hline Type of Opioid & $\begin{array}{l}\text { Proportion (\%) of Administrations } \\
\text { Considered Potentially Inappropriate }\end{array}$ & $P$ Value \\
\hline Morphine & $210 / 471(44.6 \%)$ & \\
\hline Hydromorphone & $221 / 808(27.4 \%)$ & $<.001^{a}$ \\
\hline Fentanyl & $1 / 39(2.6 \%)$ & \\
\hline Methadone & $0 / 1(0 \%)$ & \\
\hline \multicolumn{3}{|c|}{$\begin{array}{l}\text { aRate of inappropriate administration was compared between morphine, hydromorphone, } \\
\text { and fentanyl administrations using a Chi-square test; methadone was excluded from the } \\
\text { comparison owing to small sample size (presence of a zero cell). }\end{array}$} \\
\hline
\end{tabular}

other, unknown, or declined were less likely to have potentially inappropriate use.

\section{DISCUSSION AND CONCLUSIONS}

In this cohort of medical and surgical inpatients, we found that almost one-third received at least one potentially inappropriate IV opioid administration during their hospitalization, and one-third of all IV opioid administrations were potentially inappropriate based on current recommendations defining the appropriate use of IV versus oral opioids. Although this is a single-center analysis, to our knowledge, this is the first study to ascertain the rate of potentially inappropriate IV opioid administration in hospitalized patients. Our findings suggest that quality improvement initiatives are necessary to promote more guideline-concordant care in this realm.

Several factors may contribute to overuse. Requests from patients for immediate pain relief may at times drive prescription of the IV formulation. In addition, patients may expect the IV formulation because of precedents from prior interactions with the healthcare system. Both of these situations may be opportunities for patient education about the equivalent bioavailability of oral and IV formulations in patients with a functioning GI tract, as well as the relatively small difference in rate of onset between the two routes of administration (generally 15-20 minutes). When a patient's pain is well controlled with IV medications, physicians may also fail to recognize the need to transition to PO medications, further prolonging unnecessary use. Finally, in patients with multiple, complex, or deteriorating medical conditions, transitioning to oral opioids may be deprioritized for the sake of addressing more urgent medical concerns.

This study highlights the potential for transitioning more patients to oral opioids, which should be feasible in the inpatient setting, where pain needs can often be anticipated in advance and oral medications can be administered earlier to overcome the short delay in the onset of action between the oral and IV routes. Oral medications also have the advantage of a longer duration of effect, which may provide overall improved pain control. At our institution, a recent shortage of IV opioids (which occurred after the data collection period for this study) and subsequent efforts to limit IV opioid use (via computerized prompts and active pharmacist consultation) resulted in an immediate $50 \%$ reduction in the daily number of IV opioid administrations, further supporting our conclusion that there is an opportunity to decrease inappropriate use of IV opioids.

There were no specific patient factors that contributed to potentially inappropriate use. Although the ethnicity category of other/unknown/declined was significantly less likely to receive opioids potentially inappropriately, given the heterogeneity of this group, it is difficult to draw conclusions on the clinical significance of this finding. Morphine was significantly more likely than other opioids to be administered inappropriately.

There are several limitations of this study. Because this was a retrospective review, our criteria for appropriate use may have resulted in some misclassification; as a result, we can comment only on potentially inappropriate use rather than on definitively inappropriate use. We attempted to use a conservative definition of appropriateness by automatically assuming all doses in the first 24 hours of administration to be appropriate, which could have resulted in underestimating potentially inappropriate use. Nonetheless, there may be instances in which a patient had suspected malabsorption that was not captured or a fluctuating ability to receive oral medications within a given 24-hour period (due to nausea, for example), resulting in outcome misclassification. In addition, we did not correlate findings with patient-reported pain scores. Because there is no clearly defined pain threshold at which IV opioids are indicated, we did not believe that would be useful in clarifying appropriate versus inappropriate use. That said, we believe that, most of the time, pain medications should be able to be titrated appropriately within 24 hours to avoid the need for immediate pain relief with IV opioids thereafter. Although there may be instances of patients who have breakthrough pain severe enough to require IV opioids despite adequate titration of oral medications, we believe this is likely to represent a small number of our population that received potentially inappropriate use. It is worth noting that even if we overestimated by $50 \%$, such that the true rate of potentially inappropriate IV administrations is $15 \%$, we believe this would still be a ripe target for quality improvement initiatives, given that tens of millions of hospitalized patients receive opioids each year in the United States. ${ }^{10}$ Finally, we were unable to quantify the number of providers involved in decision-making for these patients, and the single-center nature and short time frame of the study limit generalizability; our analysis should be replicated at other hospitals.

In conclusion, in this sample of 200 medical and surgical hospitalizations receiving IV opioids at a large academic medical center, we identified potentially inappropriate IV administration in $31 \%$, suggesting potential to improve value through improving prescribing practices.

Disclosures: None of the authors have conflicts to disclose.

Funding: Dr. Herzig is funded by grant number K23AG042459 from the National Institute on Aging and R01HS026215 from the Agency for Healthcare Research and Quality. The manuscript contents are solely the responsibility of the authors and do not necessarily represent the views of the funding organizations. 


\section{References}

1. Dowell D, Haegerich TM, Chou R. CDC guideline for prescribing opioids for chronic pain-United States, 2016. JAMA. 2016;315(15):1624-1645. https://doi. org/10.1001/jama.2016.1464

2. Herzig SJ, Mosher HJ, Calcaterra SL, Jena AB, Nuckols TK. Improving the safety of opioid use for acute noncancer pain in hospitalized adults: a consensus statement from the Society of Hospital Medicine. J Hosp Med. 2018;13(4):263-271. https://doi.org/10.12788/jhm.2980.

3. Daoust R, Paquet J, Lavigne G, Piette E, Chauny JM. Impact of age, sex and route of administration on adverse events after opioid treatment in the emergency department: a retrospective study. Pain Res Manag. 2015;20(1):23-28. https://doi.org/10.1155/2015/316275.

4. Overdyk F, Dahan A, Roozekrans M, van der Schrier R, Aarts L, Niesters M. Opioid-induced respiratory depression in the acute care setting: a compendium of case reports. Pain Manag. 2014;4(4):317-325. https://doi. org/10.2217/pmt.14.19.

5. Wang Y, Sands LP, Vaurio L, Mullen EA, Leung JM. The effects of postoperative pain and its management on postoperative cognitive dysfunction. Am J Geriatr Psychiatry. 2007;15(1):50-59. https://doi.org/10.1097/01. JGP.0000229792.31009.da.
6. Al-Qadheeb NS, O'Connor HH, White AC, et al. Antipsychotic prescribing patterns, and the factors and outcomes associated with their use, among patients requiring prolonged mechanical ventilation in the long-term acute care hospital setting. Ann Pharmacother. 2013;47(2):181-188. https://doi. org/10.1345/aph.1R521.

7. Compton WM, Volkow ND. Abuse of prescription drugs and the risk of addiction. Drug Alcohol Depend. 2006;83(1):S4-S7. https://doi.org/10.1016/j. drugalcdep.2005.10.020.

8. O'Brien CP. Drug addiction and drug abuse. In: Hardman JG, ed. Goodman and Gilman's Pharmacological Basis of Therapeutics. New York: McGraw-Hill; 2001:621-642.

9. Lau BD, Pinto BL, Thiemann DR, Lehmann CU. Budget impact analysis of conversion from intravenous to oral medication when clinically eligible for oral intake. Clin Ther. 2011;33(11):1792-1796. https://doi.org/10.1016/j. clinthera.2011.09.030.

10. Herzig SJ, Rothberg MB, Cheung M, Ngo LH, Marcantonio ER. Opioid utilization and opioid-related adverse events in nonsurgical patients in US hospitals. J Hosp Med. 2014;9(2):73-81. https://doi.org/10.1002/ jhm.2102. 